\title{
Less approach, more avoidance: Response inhibition has motivational consequences for sexual stimuli that reflect changes in affective value not a lingering global brake on behavior
}

\author{
Rachel L. Driscoll ${ }^{1}$ - Keelia Quinn de Launay ${ }^{1}$ - Mark J. Fenske ${ }^{1}$
}

Published online: 21 April 2017

(C) Psychonomic Society, Inc. 2017

\begin{abstract}
Response inhibition negatively impacts subsequent hedonic evaluations of motivationally relevant stimuli and reduces the behavioral incentive to seek and obtain such items. Here we expand the investigation of the motivational consequences of inhibition by presenting sexually appealing and nonappealing images in a go/no-go task and a subsequent image-viewing task. Each initially obscured image in the viewing task could either be made more visible or less visible by repeatedly pressing different keys. Fewer key presses were made to obtain better views of preferred-sex images when such images had previously been inhibited as no-go items than when previously encountered as noninhibited go items. This finding replicates prior results and is consistent with the possibility that motor-response suppression has lingering effects that include global reductions in all behavioral expression. However, for nonpreferred images, prior inhibition resulted in more key presses to obscure their visibility than when such images had not been inhibited. This novel finding suggests that the motivational consequences of response inhibition are not due to a global brake on action but are instead linked to negative changes in stimulus value that induce corresponding increases in avoidance and decreases in approach.
\end{abstract}

Keywords Inhibition - Stimulus value Affective evaluation Behavioral motivation

Anyone who has crossed a busy street when rushing late to an appointment can appreciate the adaptive value of suppressing a

Mark J. Fenske

mfenske@uoguelph.ca

1 Department of Psychology, University of Guelph, Guelph, Ontario, Canada N1G 2W1 prepotent behavioral action (e.g., crossing the street) when situational factors (e.g., fast-approaching vehicles) deem the action inappropriate. Mechanisms of motor-response inhibition supporting such action suppression are typically studied using behavioral tasks measuring the ability to stop an ongoing response (e.g., stop-signal task; Logan \& Cowan, 1984) or withhold from making a response to a pertinent stimulus (e.g., go/ no-go task; Donders, 1868/1969). The behavioral indices of response inhibition in these studies have been linked to neurophysiological suppression of motor activity (Sohn, Wiltz, \& Hallet, 2002), including reductions in cortico-spinal excitability and diminished motor-evoked potentials (e.g., Hoshiyama et al., 1996; Leocani, Cohen, Wassermann, Ikoma, \& Hallett, 2000). Reductions in motor-related neural activity are thereby thought to underlie reductions in the propensity to execute a specific behavior (see Chambers, Garavan, \& Bellgrove, 2009; Stinear, Coxon, \& Byblow, 2009, for review; Rubia et al., 2001; Coxon, Stinear, \& Byblow, 2006).

The corresponding impact on subsequent behavior may be linked to findings that response inhibition can enhance the ability to resist the motivational allure of stimuli that might otherwise elicit failures of self-control. Appetitive food stimuli are less likely to produce approach-motivated behavior, for instance, if they have been previously associated with stopsignal cues (e.g., Houben, Havermans, Nederkoorn, \& Jansen, 2012; Veling, Aarts, \& Stroebe, 2013). Manipulations of response-inhibition within gambling tasks can also impact the size of bets and other forms of gambling-related approach behavior (Stevens et al., 2015). Likewise, alcohol consumption can be reduced when participants withhold motor responses from alcohol images (Houben, Nederkoom, Wiers, \& Jansen, 2011; Jones \& Field, 2013), and participants are less willing to invest time and effort seeking views of sexually appealing images when such images have been associated with response inhibition (Ferrey, Frischen, \& Fenske, 2012). 
These findings underscore the possibility that suppressing a motor response leads to decreased capacity to energize subsequent behavior, even for motivationally salient stimuli that otherwise strongly promote behavioral approach (Freeman, Alvernaz, Tonnesen, Linderman, \& Aron, 2015). Indeed, neurophysiological research suggests that achieving rapid behavioral suppression is facilitated by a nonspecific reduction in global neuro-motor activity across a wide range of motor systems (Aron, 2007; Coxon et al., 2006). For example, rapid handmovement suppression, speech suppression, and eye-movement suppression have all been shown to concurrently reduce corticospinal activity and motor-evoked potentials in task-irrelevant muscles (Badry et al., 2009; Cai, Oldenkamp, \& Aron, 2012; Wessel, Reynoso, \& Aron, 2013). Although a lingering global suppression of motor activity could explain the decreased capacity of motivationally-salient stimuli to energize behavior in the immediate aftermath of the application of response inhibition, it is less clear how it would account for behavioral effects extending throughout the following week (e.g., Houben et al., 2011).

Another possibility is that the motivational consequences of response inhibition are instead due to an inhibition-related alteration of stimulus value. An individual is less likely to expend effort seeking a motivationally relevant stimulus, not because the ability to execute the necessary motor actions has been affected but because the stimulus itself has become less motivationally appealing. Several recent findings support this possible link between inhibition and stimulus devaluation, including the consistent observations that stimuli that are ignored or otherwise inhibited in selective attention (e.g., Raymond, Fenske, \& Tavassoli, 2003; Raymond, Fenske, \& Westoby, 2005; De Vito, Al-Aidroos, \& Fenske, 2017; Fenske, Raymond, \& Kunar, 2004; Fenske, Raymond, Kessler, Westoby, \& Tipper, 2005; Kiss et al., 2007; Martiny-Huenger, Gollwitzer, \& Oettingen, 2014) or memory-suppression tasks (De Vito \& Fenske, 2017; Vivas, Marful, Panagiotidou, \& Bajo, 2016), or from which a response is withheld in go/no-go (e.g., Frischen, Ferrey, Burt, Pistchick, \& Fenske, 2012; Ferrey et al., 2012; Kiss, Raymond, Westoby, Nobre, \& Eimer, 2008) or stop-signal tasks (Wessel, Tonnesen, \& Aron, 2015) subsequently receive more negative affective evaluations than the targets of attention/response. Such cognitive-behavioral and neuroimaging results have been taken as evidence that inhibition itself may alter the coding and representation of stimulus value (for reviews, see Fenske \& Raymond, 2006; Raymond, 2009; Gollwitzer, Martiny-Huenger, \& Oettingen, 2014).

\section{Current study}

Here, we test competing predictions that arise from the globalsuppression and devaluation-by-inhibition accounts of the stimulus-linked motivational consequences of response inhibition on motivationally relevant behavior. We used the same go/no-go task that Ferrey et al. (2012) used to associate response inhibition with erotic images of participants' preferred and nonpreferred sex. In a subsequent affective-evaluation task, they found that previously inhibited (no-go) items were subsequently rated as less attractive than were noninhibited (go) items, and that the magnitude of this effect was similar for both preferred-sex and nonpreferred images. Furthermore, in a motivated-viewing task, Ferrey et al. observed that prior inhibition of preferred-sex images reduced participants' willingness to expend time and keypressing effort to view such motivationally relevant stimuli. While this decrease in approach behavior toward otherwise appealing stimuli is predicted by both the global-suppression and devaluation-by-inhibition hypotheses, we modified the motivated-viewing task to provide a measure of stimulusdriven motivated behavior about which these competing hypotheses make opposite predictions. Specifically, rather than using a task that only assesses an inhibition-related change in stimulus approach, we developed a task with which we could also assess possible inhibition-related changes in participants' willingness to expend time and key-pressing effort to escape views of unwanted stimuli. This is important because an inhibition-related global brake on motor activity and lingering decrease in the ability to energize subsequent behavior predicts a reduction in all keypressing behavior regardless of whether keys are being pressed to view appealing images or to avoid viewing unappealing images. In contrast, an inhibition-related reduction of stimulus value predicts a decrease in key presses to view otherwise appealing images but an increase in key presses to avoid viewing images that have become even more unappealing through stimulus devaluation.

The inclusion of a measure of stimulus avoidance also underscores the importance of the present experiment for addressing the void in the literature concerning how response inhibition impacts processing linked to behavioral avoidance of motivationally relevant stimuli. Whereas a growing number of studies have examined the impact of appetitive and aversive stimuli on mechanisms of response inhibition (e.g., Albert, López-Martín, \& Carretié, 2010; Yuan et al., 2012) and several studies have now examined the impact of response inhibition on behavioral approach elicited by appetitive stimuli (e.g., Ferrey et al., 2012; Houben et al., 2012; Houben et al., 2011; Veling et al., 2013), nothing is known yet about the corresponding impact of response inhibition on behavioral avoidance elicited by aversive stimuli.

\section{Method}

The methods used in our experiment were a modified version of those used by Ferrey et al. (2012) and were approved by the University of Guelph Research Ethics Board (REB\#15MY034). 


\section{Participants}

One hundred and sixty undergraduate students, ages 18 to 35 years ( $M=18.56$ years, $S D=2.45$ years), with normal or corrected-to-normal vision, were recruited from the University of Guelph undergraduate research participant pool in exchange for course credit. The appropriateness of our sample size was determined using $\mathrm{G}^{*}$ Power (Faul, Erdfelder, Lang, \& Buchner, 2007), which indicated that 80 participants would be required to detect the same-sized effect of response inhibition on behavioral approach as in Ferrey et al.'s (2012) Experiment $2(d=.82)$ with a power of 0.95 at an alpha of .05 . However, we were primarily interested in an effect of response inhibition on behavioral avoidance. The existence and size of such an effect is not yet known, however, and there is some affective-rating evidence that the consequences of response inhibition may be greater for appetitive stimuli than for affectively neutral or aversive stimuli (e.g., Chen, Veling, Dijksterhuis, \& Holland, 2016; Veling, Holland, \& van Knippenberg, 2008). This supports our decision to use a sample that was twice the size determined by our formal calculation to ensure that we could detect an effect of response inhibition on behavioral avoidance, even if it were more moderate in magnitude than that for behavioral approach. One participant left the experiment shortly after it began without further explanation. Data from five participants were excluded because of low accuracy on the go/no-go task ( $>2.5$ SDs below average). Data from an additional seven participants were excluded because they indicated equal attraction to males and females. Only individuals strongly attracted to males or females were included because our hypotheses specifically related to the impact of inhibition on sexually relevant stimuli. These exclusion criteria were determined a priori based on previous work (i.e., Ferrey et al., 2012; Frischen et al., 2012; Veling et al., 2008). Results are reported for the remaining 147 participants. Thirty-nine of the 41 males within this sample reported being strongly attracted to females; 105 of the 106 females reported being strongly attracted to males.

\section{Stimuli and apparatus}

The stimulus set included 288 digital color photographs of light-skinned attractive males and females. No nudity was shown, but images were selected for their explicit sexual appeal. Half of the images were males, half were females; both sets contained an equal number of light-haired and dark-haired individuals. Each image appeared at display center on a white background, subtending approximately $10.6^{\circ} \times 16.3^{\circ}$ visual angle at a viewing distance of approximately $60 \mathrm{~cm}$. A black plus $(+)$ symbol that subtended approximately $0.90^{\circ} \times 0.90^{\circ}$ visual angle was used as a central fixation cross. Stimulus presentation and behavioral response collection were controlled by E-Prime 2.0 software (Psychology Software
Tools, Pittsburgh, PA, USA) running on an Intel Core 2 Duo computer with a $50.8 \mathrm{~cm}$ LCD monitor (resolution: $1680 \times$ 1050 pixels).

\section{Design and procedure}

Response inhibition and affective-evaluation phase The first phase combined alternating sequences of go/no-go trials and affective-evaluation trials to assess the impact of response inhibition on perceived attractiveness of preferred-sex and nonpreferred-sex images. This phase consisted of 16 blocks, each comprised of 12 go/no-go trials followed by 12 affectiveevaluation trials (see Fig. 1). The go/no-go trials required participants to press the spacebar with both index fingers as quickly as possible whenever the person depicted in the image had dark hair (or light hair; this go cue switched once halfway through the session, its order counterbalanced across participants) and to otherwise refrain from responding. Imageseach presented for $250 \mathrm{~ms}$ followed by a 1,000 ms fixation display - were randomly selected with the constraint that each set of 12 trials comprised equiprobable factorial combinations of sex and hair color. In the affective-evaluation trials, participants rated the attractiveness of the same 12 images on a 4point scale ranging from 1 (not at all attractive) to 4 (very attractive). Participants were encouraged to use the full range of responses. Images were presented in the same order for $250 \mathrm{~ms}$ each, followed by a $1,000-\mathrm{ms}$ blank display. Participants were instructed to indicate the attractiveness of the person in each image based on their immediate personal reaction rather than an estimate of objective attractiveness.

Motivated-viewing phase The second phase of the study used a progressive-ratio key-press task to assess the impact of prior inhibition on the capacity of preferred-sex and nonpreferred images to elicit behavioral approach or avoidance (see Fig. 2). This phase consisted of eight blocks of 12 image-viewing trials. Each image of an attractive male or female initially appeared partially obscured by a moderate amount of random colored noise applied with a uniform distribution. Participants were told that they could press a key labeled clear to see more of an image or a different key labeled blurry to see less of an image. The keys were on a progressiveratio schedule such that each time an image within a given category (e.g., male images) was made completely clear or completely obscured, twice as many key presses were needed to make the next image from that category completely clear or completely obscured. Participants were instructed that any key-press response was acceptable, but that they eventually needed to reach one of the extremes (i.e., fully clear or fully obscured) in order to progress to the next image. Individuals could make up to 32 key presses to see a certain type of image (male or female); however, if at any point they changed their mind, they could make the opposite response. 
Levels of approach motivation for preferred-sex and nonpreferred images were operationalized as the number of key presses a participant made to completely enhance the visibility of the images from that category. Levels of avoidance motivation for preferred-sex and nonpreferred images were operationalized as the number of key presses a participant made to completely obscure the visibility of images from that category.

Questionnaires and debriefing Following both phases, participants filled out a demographic questionnaire, which included a modified Kinsey scale to assess self-reported sexual attraction (Kinsey Institute, Indiana University). This 7-point scale asked participants to mark the description that best described their sexuality, from exclusively attracted to females to exclusively attracted to males and included an option to indicate no socio-sexual contacts or reactions. All participants were then debriefed before leaving the experiment.

\section{Results}

Because each participant experienced only one particular combination of response-status and sexual-relevance conditions (i.e., preferred-sex images on no-go trials and nonpreferred images on go trials, or vice versa), the impact of response inhibition on each of our dependent measures (hedonic ratings and approach- and avoidance-motivated key presses to erotic images) had to be assessed between groups. We did this using two-way ANOVAs that treated both response-status (go vs. no-go) and sexual-relevance (preferred vs. nonpreferred sex) as between-subjects factors. Independent-samples $t$ tests (two-tailed) were used for planned comparisons involving simple effects. Ratings from go/no-go trials in which an error was made $(M=4.6 \%, S D=$ 3.7) were excluded from analysis.

\section{Hedonic ratings}

Participants rated preferred-sex images $(M=2.94)$ as significantly more attractive than nonpreferred images $(M=1.97)$, $F(1,290)=223.94, p<.001, \eta^{2}=.44$, confirming their sexually appealing nature. Furthermore, participants rated no-go images as significantly less attractive than go images, $F(1$, $290)=10.54, p=.001, \eta^{2}=.04$; this was the case for both the preferred-sex, $t(145)=2.40, p=.018, d=.50$, and the nonpreferred conditions, $t(145)=2.20, p=.029, d=.40$; see Fig. 3. There was no significant interaction between these effects $(F<1)$. This pattern of results showing that both sexually appealing and nonappealing stimuli become hedonically devalued after being associated with response inhibition closely replicates the same findings by Ferrey et al. (2012).

\section{Motivated-viewing key presses}

Stimulus-approach motivation The number of times participants pressed the clear key to obtain better views of preferred-sex images $(M=17.68)$ was significantly greater than that for nonpreferred images $(M=3.60), F(1,290)=$ $290.30, p<.001, \eta^{2}=.50$, confirming their motivational capacity to elicit behavioral approach. Furthermore, the number of times participants pressed the clear key to obtain better views decreased significantly when such images had previously appeared as no-go items $(M=9.18)$ than as go items $(M=12.18), F(1,290)=12.48, p<.001, \eta^{2}=.04$; this was the case for the preferred-sex, $t(145)=3.09, p=.002, d=.51$, but only marginally so for the nonpreferred condition, $t(145)=$ $1.72, p=.087, d=.30$; see Fig. 4 . The interaction between these effects was also only marginally significant, $F(1,290)=$ $3.11, p=.079, \eta^{2}=.01$. Finding that the motivational capacity of sexually appealing images to elicit behavioral approach is diminished after being associated with response inhibition also closely replicates the same findings by Ferrey et al. (2012).

Stimulus-avoidance motivation The number of times participants pressed the blurry key to escape views of nonpreferred sex images $(M=14.91)$ was significantly greater than that for preferred-sex images $(M=1.89), F(1,290)=255.72, p<.001$, $\eta^{2}=.50$, confirming their motivational capacity to elicit behavioral avoidance. Furthermore, the number of times participants pressed the blurry key to escape views increased significantly when such images had previously appeared as nogo items $(M=9.18)$ than as go items $(M=7.07), F(1,290)=$ $10.70, p=.001, \eta^{2}=.04$; this was the case for the nonpreferred sex, $t(145)=2.95, p=.004, d=.50$, but not the preferred-sex condition, $t(145)=1.64, p=.103, d=.30$; see Fig. 4. The interaction between these effects was significant, $F(1,290)=$ $6.00, p=.015, \eta^{2}=.02$. Finding that the motivational capacity of sexually unappealing images to elicit behavioral avoidance is enhanced after being associated with response inhibition is a novel and important discovery.

\section{Discussion}

Response inhibition reduces the capacity of visual stimuli to elicit approach-motivated behavior (e.g., Ferrey et al., 2012; Freeman et al., 2015; Houben et al., 2012; Jones \& Field, 2013; Veling et al., 2013; Wessel et al., 2015), but it remains unclear exactly how this occurs: through a modulation of stimulus value as predicted by devaluation-by-inhibition or through a lingering brake on behavior as predicted by global suppression. We directly compared and contrasted the globalsuppression and devaluation-by-inhibition accounts of the motivational consequences of response inhibition by modifying Ferrey et al.'s (2012) procedure to not only provide a 
measure of inhibition-related change in hedonic value and motivated stimulus approach but to also include a measure of inhibition-related change in motivated stimulus avoidance. Our results provide strong support for the devaluation-byinhibition hypothesis.

The global-suppression hypothesis predicts a reduction in all key-pressing behavior regardless of whether keys are being pressed to obtain views of appealing images or to avoid viewing unappealing images. In contrast, the devaluation-byinhibition hypothesis predicts a decrease in key presses to obtain views of otherwise appealing images but an increase in key presses to avoid viewing images that have become even more unappealing through devaluation. This is precisely what we found: Participants were less willing to repeatedly press a key to obtain better views of sexually appealing images after being associated with response inhibition, but they worked harder to escape views of sexually unappealing images after being associated with response inhibition. Such inhibitionrelated increases in behavioral expression are inconsistent with a lingering global brake on action.

Our findings closely replicate those reported by Ferrey et al. (2012) concerning the impact of response inhibition on the hedonic value and motivational salience of sexual stimuli. This includes the finding that both sexually appealing and nonappealing images received more negative hedonic evaluations after being associated with response inhibition on no-go trials than with response on go trials. These hedonicevaluation findings converge nicely with prior evidence that both positively valenced and negatively valenced stimuli receive more negative affective ratings after being associated with response inhibition on no-go trials than with response on go trials (e.g., Frischen et al., 2012). This evidence is consistent with the interpretation that inhibition does not merely attenuate the intensity of stimulus-evoked emotion linked to an item's existing affective status but has a distinctly negative impact on stimulus value, per se. Our close replication of Ferrey et al.'s (2012) results also includes the finding that the motivational incentive to seek and obtain views of otherwise appealing sexual images is significantly reduced in individuals who have recently inhibited images of that type. This finding is noteworthy for showing that a reduction in motivational incentive carries over to different instances of the inhibited stimulus category, and extends prior observations that inhibition-related reductions in hedonic value can generalize to influence evaluations of other stimuli, as long as they are from the same category or otherwise share the same features as previously inhibited items (e.g., Goolsby et al., 2009; Raymond et al., 2003).

While the replication of key experimental findings is important, the truly novel and theoretically meaningful finding from our experiment is that such generalized effects of response inhibition are not limited to reductions in motivated approach of appetitive stimuli but also include increases in motivated avoidance of aversive stimuli. This finding helps to address a void in the literature concerning how response inhibition impacts processing linked to behavioral avoidance of motivationally relevant stimuli. Whereas a growing number of studies have examined the impact of appetitive and aversive stimuli on mechanisms of response inhibition (e.g., Albert et al., 2010; Yuan et al., 2012), and several studies have examined the impact of response inhibition on behavioral approach elicited by appetitive stimuli (e.g., Ferrey et al., 2012; Houben et al., 2012; Houben et al., 2011; Veling et al., 2013), the results from our motivated-viewing task with sexually unappealing images represent the first advance in knowledge about the corresponding impact of response inhibition on the motivational capacity of stimuli to elicit behavioral avoidance. The convergence in findings that stimulus-associated response inhibition can negatively impact hedonic ratings of such items, reduce their capacity to elicit behavioral approach, and increase their capacity to elicit behavioral avoidance suggests that changes in both subjective evaluations and motivated behavior may be linked to changes in representations of stimulus value common to both processes.

As a final note, there is growing evidence that the changes in stimulus value observed following tasks that involve attentional (e.g., Fenske et al., 2004; Fenske et al., 2005; Kiss et al., 2007; Martiny-Huenger et al., 2014; Raymond et al., 2003; Raymond et al., 2005), response (e.g., Ferrey et al., 2012; Frischen et al., 2012; Kiss et al., 2008), and memory-related inhibition (De Vito \& Fenske, 2017; Vivas et al., 2016) may be due to the affective consequences of inhibition per se. However, it is important to emphasize that our combination of go/no-go plus hedonic-rating and motivated-viewing tasks did not include a novel baseline condition against which the potential negative effects of response inhibition on the value of stimuli associated with no-go trials could be distinguished from any positive effects of active responding on the value of stimuli associated with go trials. Evidence of the enhancement of stimulus value through active responding has so far been limited (e.g., Schonberg et al., 2014; but cf. Veling et al., 2008) and possibly restricted to certain conditions that encourage rapid responses on go trials (Chen et al., 2016). Our experimental procedure did not include such conditions.

Nevertheless, Veling et al.'s (2008) behavior-stimulus interaction theory and related findings by Chen et al. (2016) raise the question of whether the processes linked to response inhibition on no-go trials and those linked to responding on go trials have the same affective consequences for positively valenced stimuli associated with behavioral approach as they do for negatively valenced stimuli associated with behavioral avoidance. The inhibition of behavioral approach on no-go trials, according to this view, specifically reduces motivational value for appetitive stimuli but not for aversive or affectively neutral items. Our finding of a significant decrease in key presses to increase visibility of preferred-sex images but only 
a marginal decrease for nonpreferred images (see Fig. 4) is consistent with this perspective. Caution is needed when interpreting this apparent interaction, however, given that it was only marginally significant and given the likelihood of it arising via a floor effect (i.e., it is hard to reduce a participant's willingness to press a key to see more of an aversive image if they already lack any motivation to press a key to view it).

The counterpart to an affectively negative impact of response inhibition selective to appetitive stimuli is the possibility of positive impact of active responding that is selective to nonappetitive stimuli. Accordingly, creating behavioral-approach associations with otherwise neutral or aversive stimuli by responding to them on go trials would enhance their motivational value more than would be expected for stimuli already associated with behavioral approach. Existing evidence of enhancement of stimulus value through active responding casts doubt on this latter possibility, however, as it includes significantly greater enhancement for stimuli with higher preexisting levels of motivational value than for lowvalue items (Schonberg et al., 2014).

Our results here and in prior work from our lab using both positively valenced and negatively valenced stimuli (Ferrey et al., 2012; Frischen et al., 2012) consistently show robust differences in hedonic ratings between stimuli associated with no-go trials and those associated with go trials, regardless of a priori affective status. We also find that the capacity of both appetitive and aversive stimuli to elicit motivated behavior is impacted by being associated with no-go versus go trials. We have taken these differences in hedonic value and motivational salience as evidence that response inhibition is linked to stimulus devaluation. However, the extent to which such differences are solely due to the affective consequences of inhibition, rather than a combination of inhibition-linked negative affect for some stimuli and active responding-linked positive affect for other stimuli, remains a question that can only be unequivocally resolved in future research that incorporates the necessary baseline conditions (e.g., Chen et al., 2016). Importantly, the need for such future research does not detract from our ability to conclude from the present results that alterations in the motivational impact of sexual stimuli encountered in response-inhibition tasks reflect changes in affective value and not a lingering global brake on behavior.

Acknowledgements This research was supported by a scholarship to R.L.D. from the Social Sciences and Humanities Research Council of Canada, and grants to M.J.F. from the Natural Science and Engineering Research Council of Canada, the Canada Foundation for Innovation, and the Ontario Ministry of Research and Innovation. We are grateful for the programming assistance of David De Vito.

\section{Appendix}

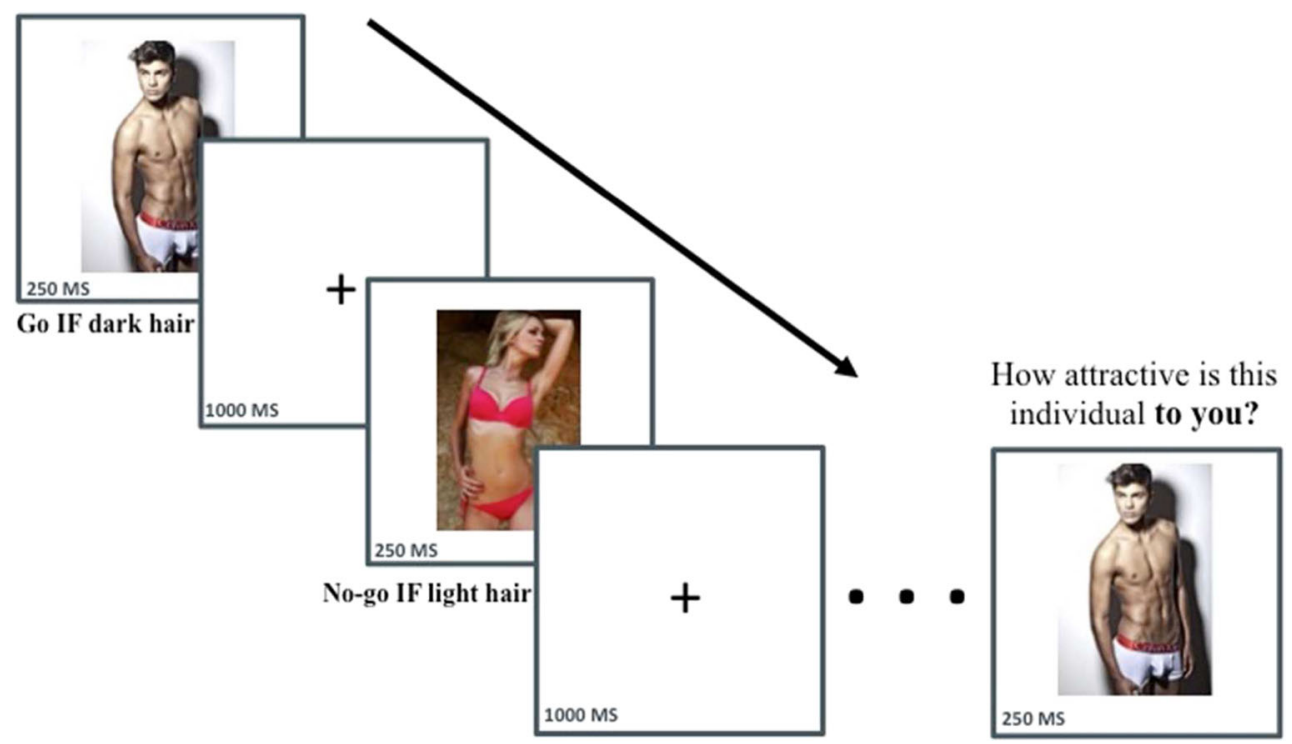

Fig. 1 Example of the stimulus sequence in the response-inhibition and affective-evaluation phase. In this example, attractive male images are go stimuli that require a speeded key-press response, and attractive female images are no-go stimuli that require the response to be withheld. After every 12 go/no-go trials, the same stimuli are affectively evaluated. (Color figure online) 


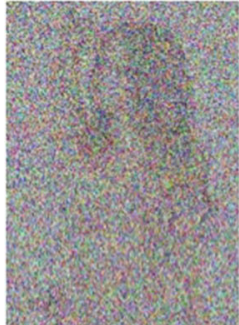

32 Presses

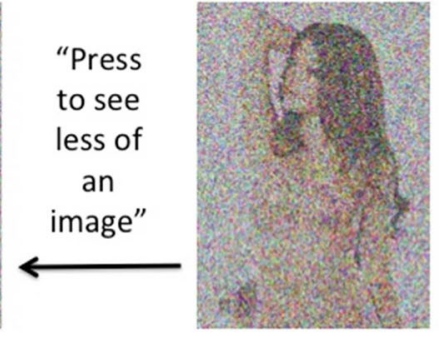

$\begin{array}{lll}1 & 2 & 4\end{array}$

Fig. 2 Example of the possible stimulus transitions in each trial of the motivated-viewing phase. Each attractive female and attractive male image initially appeared partially obscured by visual noise. Participants could choose to either make the image more visible (stimulus approach)

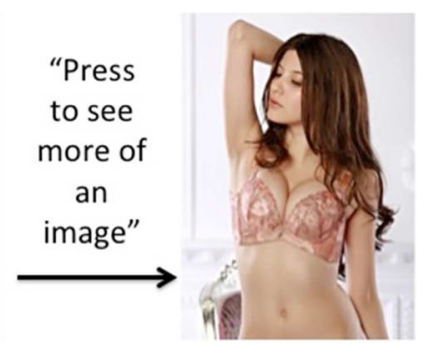

816

32

or less visible (stimulus avoidance) by pressing different keys. The keys were on a progressive-ratio schedule: twice as many presses were needed to achieve a completely clear or a complete obscured view for each successive image of a given type. (Color figure online)

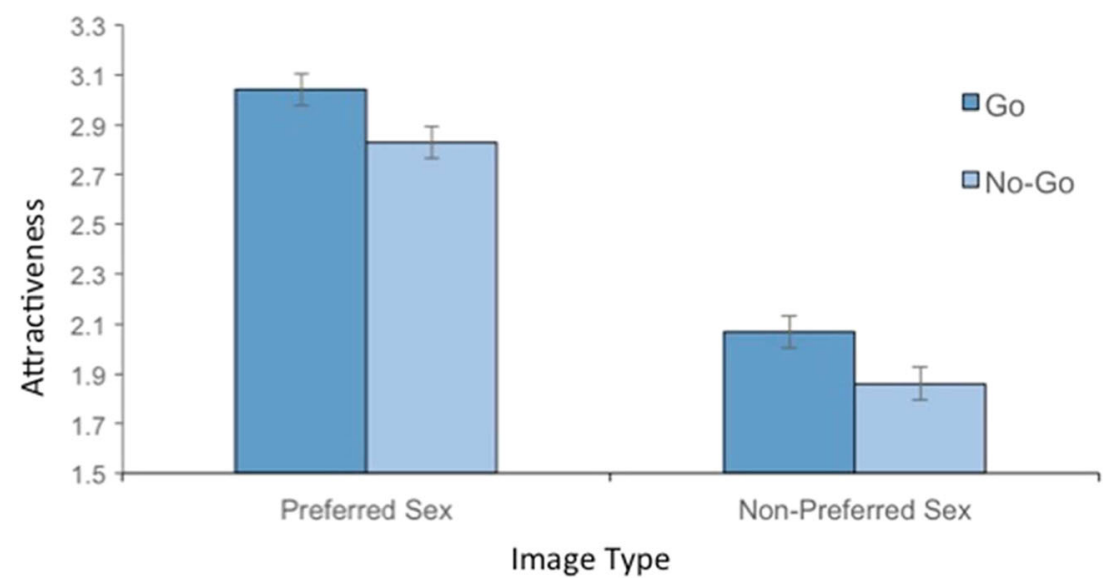

Fig. 3 Phase 1 results. Average hedonic ratings for preferred and nonpreferred stimuli according to trial type (go/no-go)

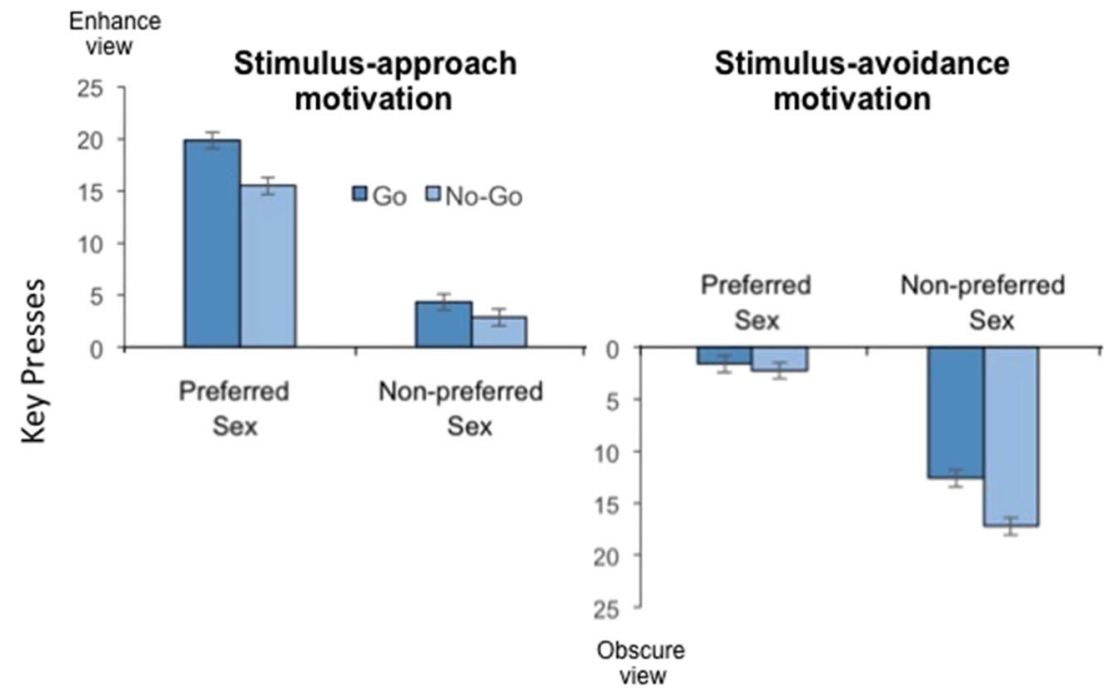

Fig. 4 Phase 2 results. Number of key presses made to view preferred stimuli or to avoid nonpreferred stimuli according to trial type (go/no-go) 


\section{References}

Albert, J., López-Martín, S., \& Carretié, L. (2010). Emotional context modulates response inhibition: Neural and behavioral data. NeuroImage, 49, 914-921.

Aron, A. R. (2007). The neural basis of inhibition in cognitive control. Neuroscientist, 13(3), 214-228.

Badry, R., Mima, T., Aso, T., Nakatsuka, M., Abe, M., Fathi, D., ... \& Fukuyama, H. (2009). Suppression of human cortico-motoneuronal excitability during the Stop-signal task. Clinical Neurophysiology, 120, 1717-1723.

Cai, W., Oldenkamp, C. L., \& Aron, A. R. (2012). Stopping speech suppresses the task-irrelevant hand. Brain \& Language, 120, 412 415 .

Chambers, C. D., Garavan, H., \& Bellgrove, M. A. (2009). Insights into the neural basis of response inhibition from cognitive clinical neuroscience. Neuroscience and Behavioural Reviews, 33, 631-646.

Chen, Z., Veling, H., Dijksterhuis, A., \& Holland, R. W. (2016). How does not responding to appetitive stimuli cause devaluation: Evaluative conditioning or response inhibition? Journal of Experimental Psychology: General, 145(12), 1687-1701.

Coxon, J. P., Stinear, C. M., \& Byblow, W. D. (2006). Intracortical inhibition during volitional inhibition of prepared action. Journal of Neurophysiology, 95, 3371-3383.

De Vito, D., Al-Aidroos, N., \& Fenske, M. J. (2017). Neural evidence that inhibition is linked to the affective devaluation of distractors that match the contents of working memory. Neuropsychologia, 99, 259-269.

De Vito, D., \& Fenske, M. J. (2017). Suppressing memories of words and familiar objects results in their affective devaluation: Evidence from think/no-think tasks. Cognition, 162, 1-11.

Donders, F. C. (1969). On the speed of mental processes. Acta Psychological, 30, 412-431 (Original work published 1868).

Faul, F., Erdfelder, E., Lang, A.-G., \& Buchner, A. (2007). G*Power 3: A flexible statistical power analysis program for the social, behavioural, and biomedical sciences. Behaviour Research Methods, 39, 175191.

Fenske, M. J., \& Raymond, J. E. (2006). Affective influences of selective attention. Current Directions in Psychological Science, 15, 312316.

Fenske, M. J., Raymond, J. E., Kessler, K., Westoby, N., \& Tipper, S. P. (2005). Attentional inhibition has social-emotional consequences for unfamiliar faces. Psychological Science, 16, 753-758.

Fenske, M. J., Raymond, J. E., \& Kunar, M. A. (2004). The affective consequences of visual attention in preview search. Psychonomic Bulletin and Review, 11, 1034-1040.

Ferrey, A. E., Frischen, A., \& Fenske, M. J. (2012). Hot or not: Response inhibition reduces the hedonic value and motivational incentive of sexual stimuli. Frontiers in Psychology, 3, 1-7.

Freeman, S. M., Alvernaz, D., Tonnesen, A., Linderman, D., \& Aron, A. R. (2015). Suppressing a motivationally-triggered action tendency engages a response control mechanism that prevents future provocation. Neuropsychologia, 68, 218-231.

Frischen, A., Ferrey, A. E., Burt, D., Pistchick, M., \& Fenske, M. J. (2012). The affective consequences of inhibition: Devaluation or neutralization? Journal of Experimental Psychology: Human Perception and Performance, 38, 169-179.

Gollwitzer, P. M., Martiny-Huenger, T., \& Oettingen, G. (2014). Affective consequences of intentional action control. In A. J. Elliot (Ed.), Advances in motivation science (Vol. 1, pp. 49-77). San Diego: Academic Press.

Goolsby, B. A., Shapiro, K. L., Silvert, L., Kiss, M., Fragopanagos, N., Taylor, J.G., ... \& Raymond, J. E. (2009). Feature-based inhibition underlies the affective consequences of attention. Visual Cognition, 17(4), 500-530.
Hoshiyama, M., Koyama, S., Kitamura, Y., Shimojo, M., Watanabe, S., \& Kakigi, R. (1996). Effects of judgement process on motor evoked potentials in go/no-go hand movement task. Neuroscience Research, 24, 427-430.

Houben, K., Havermans, R. C., Nederkoorn, C., \& Jansen, A. (2012). Beer a no-go: Learning to stop responding to alcohol cues reduces alcohol intake via reduced affective associations rather than increased response inhibition. Addiction, 107(7), 1280-1287.

Houben, K., Nederkoom, C., Wiers, R. W., \& Jansen, A. (2011). Resisting temptation: Decreasing alcohol-related affect and drinking behaviour by training response inhibition. Drug and Alcohol Dependence, 116, 132-136.

Jones, A., \& Field, M. (2013). The effects of cue-specific training on alcohol consumption in heavy social drinkers. Experimental and Clinical Psychopharmacology, 21(1), 8-16.

Kiss, M., Goolsby, B. A., Raymond, J. E., Shapiro, K. L., Silvert, L., Nobre, A.C., ... \& Eimer, M. (2007). Efficient attentional selection predicts distractor devaluation: Event-related potential evidence for a direct link between attention and emotion. Journal of Cognitive Neuroscience, 19(8), 1316-1322.

Kiss, M., Raymond, J. E., Westoby, N., Nobre, A. C., \& Eimer, M. (2008). Response inhibition is linked to emotional devaluation: Behavioural and electrophysiological evidence. Frontiers in Human Neuroscience, 2, 1-9.

Leocani, L., Cohen, L. G., Wassermann, E. M., Ikoma, K., \& Hallett, M. (2000). Human corticospinal excitability evaluated with transcranial magnetic stimulation during different reaction time paradigms. Brain, 123, 1161-1173.

Logan, G. D., \& Cowan, W. B. (1984). On the ability to inhibit thought and action: A theory of an act of control. Psychological Review, 91, 295-327.

Martiny-Huenger, T., Gollwitzer, P. M., \& Oettingen, G. (2014). Selective attention to in- and out-group members systematically influences intergroup bias. Social Psychological and Personality Science, 5(8), 936-943.

Raymond, J. (2009). Interactions of attention, emotion and motivation. Progress in Brain Research, 176, 293-308.

Raymond, J. E., Fenske, M. J., \& Tavassoli, N. T. (2003). Selective attention determines emotional responses to novel visual stimuli. Psychological Science, 14, 537-542.

Raymond, J. E., Fenske, M. J., \& Westoby, N. (2005). Emotional devaluation of distracting stimuli: A consequence of attentional inhibition during visual search? Journal of Experimental Psychology: Human Perception and Performance, 31, 1404-1415.

Rubia, K., Russell, T., Overmeyer, S., Brammer, M. J., Bullmore, E. T., Sharma, T., Simmons, A., ... \& Taylor, E. (2001). Mapping motor inhibition: Conjunctive brain activations across different versions of go/no-go and stop tasks. Neurolmage, 13, 250-261.

Schonberg, T., Bakkour, A., Hover, A. M., Mumford, J. A., Nagar, L., Perez, J., \& Poldrack, R. A. (2014). Changing value through cued approach: An automatic mechanism of behavior change. Nature Neuroscience, 17(4), 625-630.

Sohn, Y. H., Wiltz, K., \& Hallet, M. (2002). Effect of volitional inhibition on cortical inhibitory mechanisms. Journal of Neurophysiology, 88 , 333-338.

Stevens, T., Brevers, D., Chambers, C. D., Lavric, A., McLaren, I. P., Mertens, M., ... \& Verbruggen, F. (2015). How does response inhibition influence decision making when gambling? Journal of Experimental Psychology: Applied, 21(1), 15.

Stinear, C. M., Coxon, J. P., \& Byblow, W. D. (2009). Primary motor cortex and movement prevention: Where stop meets go. Neuroscience and Biobehavioral Reviews, 33, 662-673.

Veling, H., Aarts, H., \& Stroebe, W. (2013). Stop signals decrease choices for palatable foods through decreased food evaluation. Frontiers in Psychology, 4, 875. 
Veling, H., Holland, R. W., \& van Knippenberg, A. (2008). When approach motivation and behavioural inhibition collide: Behavior regulation through stimulus devaluation. Journal of Experimental Social Psychology, 44, 1013-1019.

Vivas, A. B., Marful, A., Panagiotidou, D., \& Bajo, T. (2016). Instruction to forget leads to emotional devaluation. Cognition, 150, 85-91.

Wessel, J. R., Reynoso, H. S., \& Aron, A. R. (2013). Saccade suppression exerts global effects on the motor system. Journal of Neurophysiology, 110, 883-890.
Wessel, J. R., Tonnesen, A. L., \& Aron, A. R. (2015). Stimulus devaluation induced by action stopping is greater for explicit value representations. Frontiers in Psychology, 6, 1640.

Yuan, J., Meng, X., Yang, J., Yao, G., Hu, L., \& Yuan, H. (2012). The valence strength of unpleasant emotion modulates brain processing of behavioral inhibitory control: Neural correlates. Biological Psychology, 89, 240-251. 